\title{
Complex Fragment Emission From Low Energy Compound Nucleus Decay To Multifragmentation
}

\author{
L.G. Moretto, K.X. Jing, L. Phair, K. Tso and G.J. Wozniak
}

Nuclear Science Division, Lawrence Berkeley Laboratory University of California, Berkeley, California 94720, USA

Lectures given at the International School, Workshop for Young

Physicists: Relativistic Heavy Ion Physics

Prague, Czech Republic

September, 1994

This work was supported by the Director, Office of Energy Research Division of Nuclear Physics of the Office of High Energy and Nuclear Physics of the U.S. Department of Energy under Contract DE-AC03-76SF00098 


\section{DISCLAIMER}

This report was prepared as an account of work sponsored by an agency of the United States Government. Neither the United States Government nor any agency thereof, nor any of their employees, make any warranty, express or implied, or assumes any legal liability or responsibility for the accuracy, completeness, or usefulness of any information, apparatus, product, or process disclosed, or represents that its use would not infringe privately owned rights. Reference herein to any specific commercial product, process, or service by trade name, trademark, manufacturer, or otherwise does not necessarily constitute or imply its endorsement, recommendation, or favoring by the United States Government or any agency thereof. The views and opinions of authors expressed herein do not necessarily state or reflect those of the United States Government or any agency thereof. 


\section{DISCLAIMER}

Portions of this document may be illegible in electronic image products. Images are produced from the best available original document. 


\title{
Complex Fragment Emission from Low Energy Compound Nucleus Decay to Multifragmentation
}

\author{
L. G. Moretto, K. X. Jing, L. Phair, K. Tso and G. J. Wozniak \\ Nuclear Science Division, Lawrence Berkeley Laboratory, \\ University of California, Berkeley, California 94720
}

\begin{abstract}
In the first of these lectures, the experimental emission probabilities of complex fragments by low energy compound nuclei and their dependence upon energy and $Z$ value are compared to the transition state rates. In the second part, the high energy multi-fragment emission probabilities are shown to be reducible to the single fragment emission probability through the binomial distribution. The extracted one-fragment emission probabilities have a thermal dependence of the form $p=e^{-B / T}$. This suggests that multifragmentation is a sequence of thermal binary decays.
\end{abstract}

\section{A. Transition State Rates and Complex Fragment Decay-Widths}

The rates for fission decay, as well as for chemical reactions, are calculated most often by means of the transition state method[1]. In this approach, the reaction rate is equated to the flux of phase space density across a "suitably" located hyperplane normal to the "reaction coordinate". The "suitable" location is typically chosen at a saddle point in collective coordinate space, which corresponds to a bottleneck in phase space. A smart choice of the transition state location should minimize the number of phase space trajectories doubling back across the hyperplane.

The surprising success of the transition state method has prompted attempts to justify its validity in a more fundamental way, and to identify regimes in which deviations might be expected[2-4]. In what follows we shall compare experimental decay rates for complex fragment emission with transition state predictions, and search for energy $E$ and atomic number $\mathrm{Z}$ dependent deviations that can be expected to exist.

The transition state expression for the fission decay width is:

$$
\Gamma_{f}=\frac{1}{2 \pi \rho(E)} \int \rho^{*}\left(E-B_{f}-\varepsilon\right) d \varepsilon \approx \frac{T_{f}}{2 \pi} \frac{\rho^{*}\left(E-B_{f}\right)}{\rho(E)},
$$

where $\rho(E)$ is the level density of the compound nucleus, $\rho^{*}\left(E-B_{f}-\varepsilon\right)$ is the level density at the saddle point, $B_{f}$ is the fission barrier, $\varepsilon$ is the kinetic energy over the saddle along the fission coordinate and $1 / T_{f}=\partial\left[\ln \rho^{*}(x)\right] /\left.\partial x\right|_{E-B_{f}}$.

For the one dimensional case in which the only degree of freedom treated explicitly is the reaction coordinate, the decay width takes the form:

$$
\Gamma_{f}=\hbar \omega \frac{\rho^{*}\left(E-B_{f}\right)}{\rho^{*}(E)}=\hbar \omega e^{-B_{f} / T},
$$


where $T$ is the temperature of the transition state. Now both level densities correspond to the same number of degrees of freedom. The quantity $\hbar \omega$ is the oscillator phonon associated

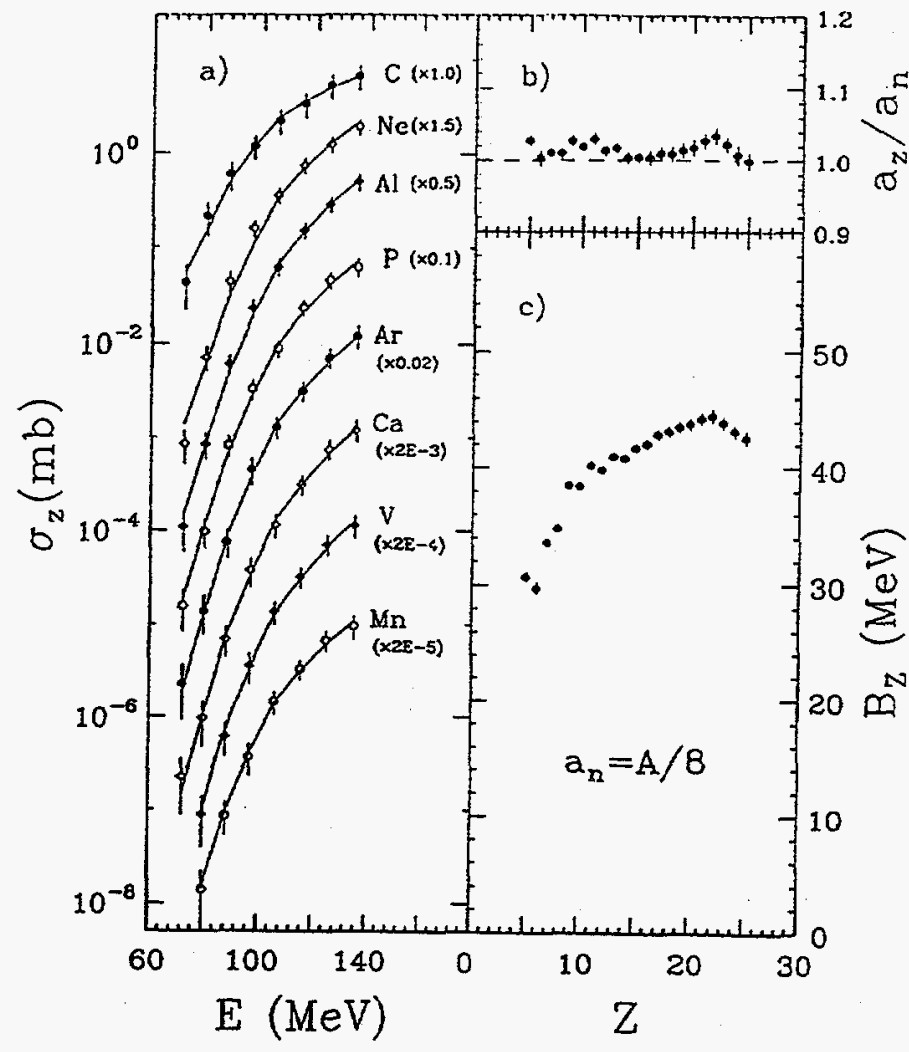

Fig. 1. a) The excitation functions (cross sections vs excitation energy) for complex fragments of some typical $Z$ values emitted from the compound nucleus ${ }^{94} \mathrm{Mo}$ produced in the reaction ${ }^{82} \mathrm{Kr}+{ }^{12} \mathrm{C}$ at beam energies ranging from 6.2 to $12.2 \mathrm{MeV} / \mathrm{u}$. b) The $a_{2} / a_{n}$ values and c) conditional barriers $B_{Z}$, both extracted by fitting the excitation functions with a transition state formalism. The solid lines in a) correspond to the fit using an energy level parameter $a_{\mathfrak{n}}=A / 8$. with the ground state minimum.

The emission of complex fragments can be treated in an analogous fashion by introducing the ridge line of conditional saddle points[5]. Each mass or charge emission can be associated with a conditional barrier. These barriers can be measured with techniques similar to those used to determine fission barriers[6]. Recently, nearly complete ridge lines have been determined for several nuclei: ${ }^{75} \mathrm{Br}[7],{ }^{90,94} \mathrm{Mo}$ [8] and $110,112 \operatorname{In}[6]$.

The emission rate of a fragment of a given mass or charge can still be described by an expression similar to that of Eq. (2). The quantity $B_{f}$ becomes the conditional barrier $B_{Z}$; but what is now the meaning of $\hbar \omega$ ? Is there a single value of $\hbar \omega$ for all the channels or has each channel its own characteristic frequency? We shall endeavor to answer this question experimentally.

An additional aspect of the problem has been studied by Kramers in his seminal work[2]. Kramers considered the diffusion of the system from the reactants' region to the products' region from the point of view of the Fokker-Planck equation. The new parameter entering the problem is the viscosity coefficient, which couples the reaction coordinate to the heat bath. The

stationary current solution found by Kramers leads to expressions for the reaction rates similar to that of the transition state theory, differing only in the pre-exponential factor, which now includes the viscosity. More recent work on the same equation has shown that if the system is forced to start at time $t=0$ at the ground state minimum, a transient time $\tau_{f}$ exists during which the reaction rate goes from zero to its stationary value[3]. Both effects would decrease the overall fission rate compared to the transition state prediction.

These effects have been advocated as an explanation for the large number of prescission neutrons observed in the fission of many systems[9-13], in apparent contradiction with the predictions of the transition state method[11, 12]. The prescission neutrons can be emitted either before the system reaches the saddle point, or during the descent from saddle to scission. Only the former component, however, has any bearing on possible deviations of the fission rate from its transition state value, and the separation of the two components is very difficult indeed. 


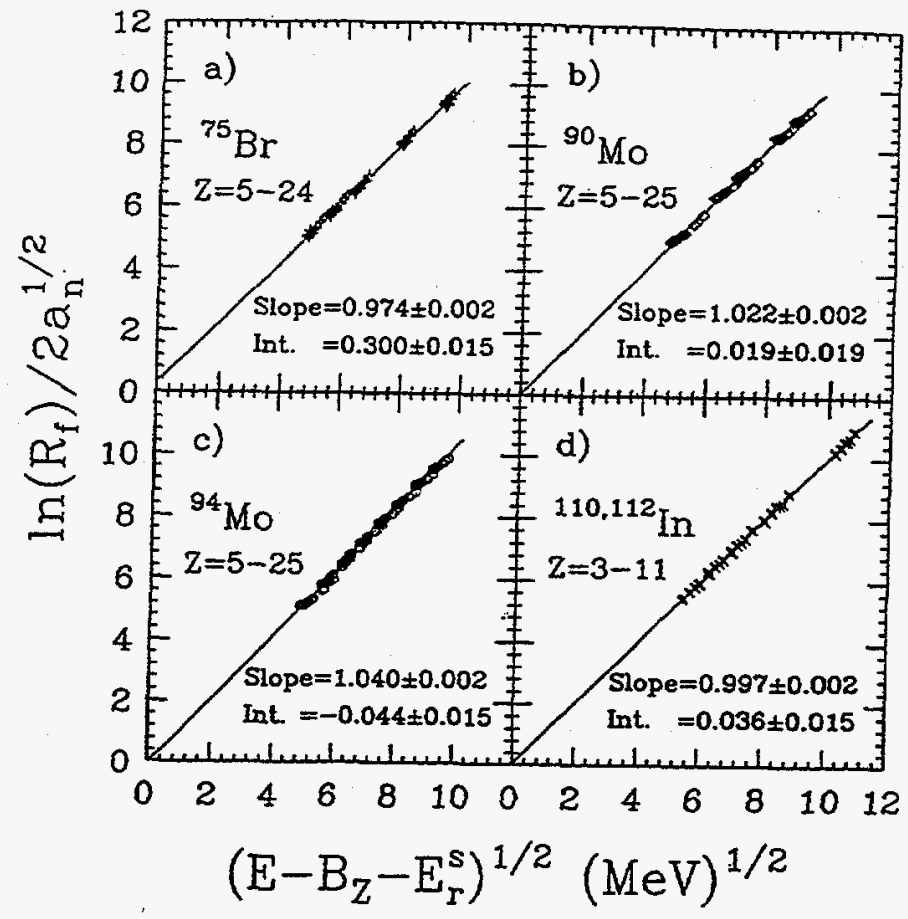

Fig. 2. $\ln R_{f} / 2 a_{n}{ }^{1 / 2}$ (see Eq. 5 and text) vs the square root of the intrinsic excitation energy for four compound nuclei: ${ }^{75} \mathrm{Br}$ a), $\left.{ }^{90} \mathrm{Mo} \mathrm{b}\right),{ }^{94} \mathrm{Mo} \mathrm{c}$ ), and 110,112 In d). All the excitation functions for the indicated $Z$ range are included for each compound nucleus. The solid lines are the linear fits to the data. The error bars are smaller than the size of symbols.
Furthermore, it has been suggested that the viscosity and the transient time may depend on the collectivity of the reaction coordinate[14]. More specifically, the reaction coordinate for a very asymmetric decay should have little collectivity, while that for a symmetric decay should be very collective. A study of prescission particles as a function of the size of the emitted fragment claims to have observed such an effect $[11,14,15]$.

We are going to show that the presence or absence of the effects discussed above can be directly observable in the excitation functions for the emission of fragments with different $Z$ values. Our procedure uses the transition state prediction as a null hypothesis, and involves only the replotting of experimental data without using any specific model. The cross section for the emission of a fragment of a given $Z$ value can be written as:

$$
\sigma_{Z}=\sigma_{0} \frac{\Gamma_{Z}}{\Gamma_{T}}=\sigma_{0} \frac{\Gamma_{Z}}{\Gamma_{n}+\Gamma_{p}+\ldots},
$$

where $\sigma_{0}$ is the compound nucleus formation cross section and $\Gamma_{\tau}, \Gamma_{n}, \Gamma_{p}, \Gamma_{z}$ are the total-, neutron-, proton-, and Z-decay widths, respectively. Notice that $\Gamma_{T}$ is essentially independent of $Z$ if we confine our observations to the excitation energy region where the complex fragment emission probability is small.

We now rewrite Eq. (3) as follows:

$$
\frac{\sigma_{\mathrm{Z}}}{\sigma_{0}} \Gamma_{T} \frac{2 \pi \rho\left(E-E_{r}^{g s}\right)}{T_{\mathrm{Z}}}=\rho^{*}\left(E-B_{\mathrm{z}}-E_{r, \mathrm{z}}^{s}\right),
$$

where $T_{Z}$ is the temperature at the conditional saddle point and $E_{r}^{g s}, E_{r, Z}^{s}$ are the ground state and saddle point rotational energies. In this way, the left hand side of the equation contains the complex fragment cross section which can be measured, and other calculable quantities that do not depend on $Z$, except $T_{Z}$ which is only weakly dependent on $Z$. The right hand side contains only the level density at the conditional saddle calculated at the intrinsic excitation energy over the conditional saddle, which is calculable if the barrier height is known.

By using the standard Fermi gas level density expression, one can rewrite Eq. (4) in the following way which takes out the A-dependence of the level density:

$$
\ln \left[\frac{\sigma_{Z}}{\sigma_{0}} \Gamma_{T} \frac{2 \pi \rho\left(E-E_{r}^{g s}\right)}{T_{z}}\right] / 2 \sqrt{a_{n}}=\frac{\ln R_{f}}{2 \sqrt{a_{n}}}=\sqrt{\frac{a_{Z}}{a_{n}}\left(E-B_{Z}-E_{r}^{s}\right)},
$$




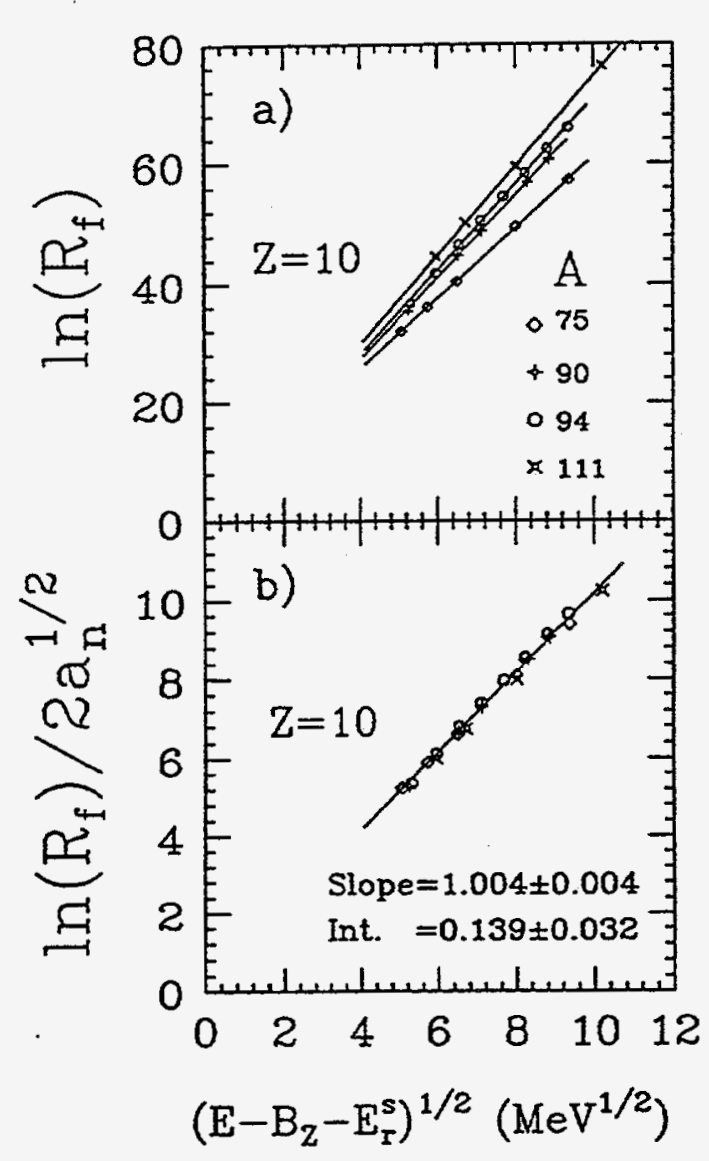

Fig. 3. In $R_{f}$ a) and $\ln R_{f} / 2 a_{n} 1 / 2$ b) vs the intrinsic excitation energy for $Z=10$ fragments from the compound nuclei: ${ }^{75} \mathrm{Br}$, ${ }^{90} \mathrm{Mo},{ }^{94} \mathrm{Mo}$, and $110,112 \mathrm{In}$. The $A$-dependence of $R_{f}$ vs $a_{n}$ is shown in a), while this $A$.

dependence is removed in b). $110,112 \mathrm{In}$, respectively. We see that all the
excitation functions for each Z-value fall with remarkable precision on the same line that is in fact straight, has a slope near unity and passes closely through zero.

The sensitivity of the excitation functions to the mass $A$ of the compound nucleus is vividly shown in the Figure $3 a \& 3 b$, where $\ln R_{f}$ is plotted vs the square root of the intrinsic excitation energy for $Z=10$ and for four different compound nuclei. The excitation functions are straight lines, but with different slopes for the different mass compound nuclei. After the $A$ dependence is removed as suggested in Eq. 5, the four lines collapse into a single straight line. Similar results are obtained for all the other $\mathrm{Z}$ values. We find this delicate sensitivity to the mass of the compound nucleus truly remarkable.

As a final virtuoso touch, we can try to collapse ALL the excitation functions for ALL $Z$ values and for all compound nuclei into a single straight line. The resulting plot for four different compound nuclei is shown in Figure 4. It includes a total of 71 excitation functions spanning a $Z$ range from 3 to 25 . The collapse of all the experimental excitation functions for all of the different $Z$-values and all the systems onto a single straight line is strong evidence for the validity of the trainsition state formalism and for the absence of $\mathrm{Z}$ - and E-dependent deviations. In particular, one is led to the following conclusions: 


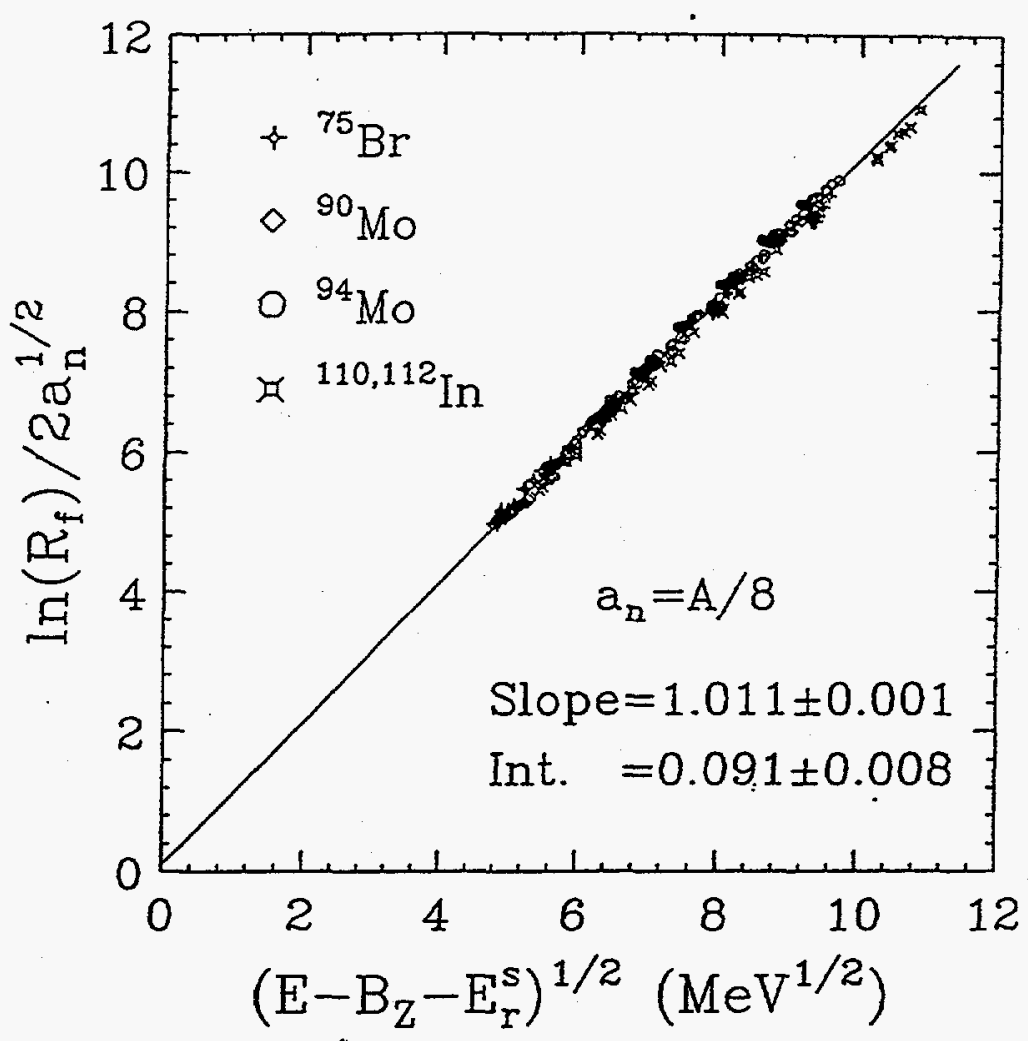

Fig. 4. The same as Fig. 2 with data for all four nuclei in a single plot. The straight line is the linear fit to all the data points

of all systems studied.

d) The intercept of the straight line, which is associated with the channel frequency $\omega$, is essentially zero and shows no obvious dependence on the fragment Z-values (i.e., the collectivity).

We conclude that in this extended data set there is no evidence for transient effects either directly or through their expected dependence upon the mass of the emitted fragment. Furthermore it appears that the channel frequency is the same for all the different $\mathrm{Z}$ decay channels.

\section{B. Time-scale and Branching Ratios in Sequential Multifragmentation}

At low excitation energies, complex fragments are emitted with low probability by a compound nucleus mechanism[16, 17]. At increasingly larger energies, the probability of complex fragment emission increases dramatically, until several fragments are observed within a single event[18-22]. The nature of this multifragmentation process is at the center of much current attention. In particular, the issue of sequentiality versus simultaneity is hotly debated theoretically[19-25], and is the object of intense experimental study[26-33].

This polarization is evident even within the framework of statistical theories. On the one hand, sequential multifragmentation theories allow the fragments present at any stage to undergo additional binary decays with probabilities determined from more or less standard compound nucleus decay widths $[5,34]$. On the other hand, chemical equilibrium-like theories 
generate fragments in chemical equilibrium, and release them simultaneously when the average density of the system falls below a critical freeze out value $[18,21,23,35,36]$.

Recent experimental work $[37,38]$ has shown that the excitation functions for the production of two, three, four, etc. fragments have a characteristically statistical energy dependence. However the issue of sequentiality versus simultaneity could not be resolved. Several efforts to settle this dispute have utilized the pairwise fragment-fragment correlations introduced by their mutual Coulomb interaction. Results have been presented showing a substantial dip in the probability of finding pairs of fragments at small relative velocities[27-30,33] and small relative angles[26, 31]. Simulations, performed with chemical equilibrium and sequential decay codes, were compared with experiment, and the rather short upper limits obtained for the decay time-scales were deemed consistent with a simultaneous break up.

A recent experiment[31] has studied the "proximity" effect of the surviving partner, produced in a deep inelastic-like collision, on the angular distribution of the fragments resulting from the break-up of the other partner. In this experiment the measured total kinetic energy loss of the primary binary collision can be related to the excitation energy of the nucleus undergoing multifragmentation. This remarkable experiment shows that at small excitation energies the "proximity" effects are essentially absent, but become very pronounced at large excitation energies. This onset of proximity effects was taken to signify a transition from "conventional" sequential multifragmentation to "true" simultaneous multifragmentation.

The conclusions drawn from these experiments are predicated upon the tacit assumption that sequential decay always occurs on a very long time scale, so that the large space-time separation of sequentially emitted fragments makes their interaction negligible. This (incorrect) assumption has been consistently incorporated into simulations based upon sequential emission codes.

In what follows we shall show three things.

First, the usual thermal binary probabilities associated with sequential emission undergo a dramatic increase with excitation energy and the corresponding emission timescale contracts dramatically, in agreement with observation[28, 29, 31, 33]. This is a very relevant, though straightforward and in many ways somewhat trivial point.

Second, the sequential time-scale and its contraction with excitation energy are directly related to the excitation functions for binary, ternary, quaternary, etc., decays through the elementary binary decay probability.

Third, we can extract this elementary binary decay probability and the corresponding time scale from the experimental excitation functions.

The first point is readily shown. For statistical decay one can rewrite the partial decay width $\Gamma$ in terms of a partial decay time $\tau$. The partial decay width associated with a given channel can be written as:

$$
\Gamma=\hbar \omega_{0} e^{-B / T},
$$

where $\omega_{o}$ is a frequency characteristic of the channel under consideration, $B$ is the barrier associated with the channel, and $T$ is the temperature. For instance, in fission $\omega_{0}$ is the collective frequency of assault on the barrier ( $\sim$ beta vibration frequency) and $B$ is the fission barrier.

The elementary probability $p$ for a binary decay to occur at any given "try" defined by the channel period $\tau_{o}=1 / \omega_{o}$ is: 


$$
p=\frac{\Gamma}{\hbar \omega_{0}}=e^{-B / T}
$$

The corresponding time is given by:

$$
\tau=\tau_{0} e^{B / T}
$$

In the case of a compound nucleus, the total decay width is the sum of the widths of all channels, and the lifetime is calculated accordingly. This lifetime defines the survival of the initial unmodified compound nucleus. For sequential multifragmentation, only the decay width and lifetime for binary fragment formation need be considered, while the abundant light particle decay can be treated as a background that may progressively modify the temperature and possibly the barrier.

Eq. 8 shows that the decay lifetime is dramatically affected even by moderate changes in temperature. Furthermore, as the temperature becomes comparable with the barrier, the binary decay probability approaches unity and the lifetime approaches the characteristic (dynamical) time constant of the channel, $\tau_{0}$.

We argue that this dramatic decrease of the decay lifetime with increasing excitation energy is the effect observed in Refs.[28, 29, 31, 33], and that this effect is inherent to the energy dependence of sequential decay, rather than a transition from sequential to true multifragmentation. If this is indeed the case, there is no need for a separate theory of multifragmentation, since this process is reducible to a sequence of binary decays that can be described in a standard way.

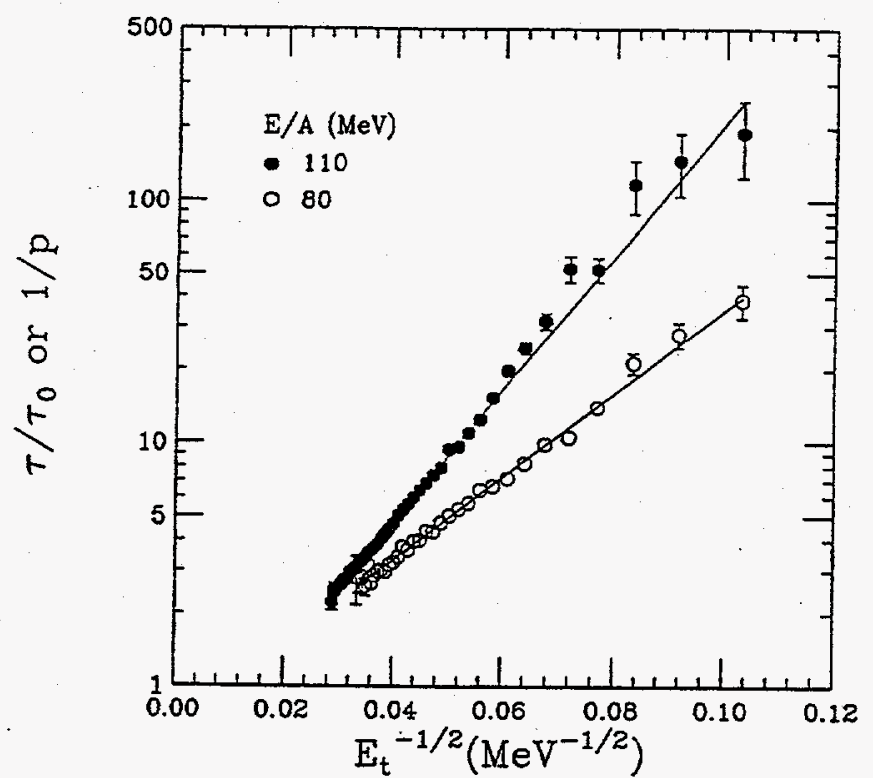

Fig. 5. The reciprocal of the binary decay probability $1 / p$ or the ratio $\tau / \tau_{0}$ (calculated from the mean and variance of the intermediate mass fragment distributions) as a function of $\mathrm{E}_{\mathrm{t}}^{-1 / 2}$ for the reaction ${ }^{36} \mathrm{Ar}+{ }^{197} \mathrm{Au}$ at $\mathrm{E} / \mathrm{A}$ at 80 (open circles) and $110 \mathrm{MeV}$ (solid circles). The solid lines are linear fits to $\log (1 / \mathrm{p})$.
As a second point, we note that the elementary binary probability $p$ (or the time $\tau$ ) can be directly related to the experimental branching ratios for binary, ternary, quaternary, etc., decay. For simplicity, let us assume that the system has the opportunity to try $m$ times to emit an "inert" fragment with constant probability $p$. The probability $P_{n}^{m}$ of emitting exactly $n$ fragments is given by the binomial distribution:

$$
P_{n}^{m}=\frac{m !}{n !(m-n) !} p^{n}(1-p)^{m-n}
$$

The average multiplicity is then

$\langle n\rangle=m p$

and the variance

$\sigma_{n}^{2}=\langle n\rangle(1-p)$ 
Thus, from the experimental values of $\langle n\rangle$ and $\sigma_{n}^{2}$ one can extract values for $p$ and $m$, at any excitation energy. Alternatively, one can extract $p$ from the ratio of any pair of excitation functions $P_{n}^{m}(T)$ :

$$
\frac{1}{p}=\frac{\tau}{\tau_{0}}=\frac{P_{n}^{m}}{P_{n+1}^{m}} \frac{m-n}{n+1}+1
$$

We now proceed to verify the above predictions by comparison with experiment. References $[39,40]$ report values of $\langle n\rangle$ and $\sigma_{n}^{2}$ for the reaction ${ }^{36} \mathrm{Ar}+{ }^{197} \mathrm{Au}$ at $80 \& 110$ $\mathrm{MeV} / \mathrm{u}$ (available center-of-mass energy of 2.4 and $3.3 \mathrm{GeV}$, respectively) as a function of the transversal energy $E_{t}$ of the event, $E_{t}=\sum \varepsilon_{i} \sin ^{2} \theta_{i}$, where $\varepsilon_{i}$ is the kinetic energy of each fragment and $\theta_{i}$ is the angle between the fragment and the beam direction. In choosing the transversal energy as our observable, we assume that it is proportional to the excitation energy $E$ of the source [41], where $E_{\imath}=K\left(E, A_{p}, A_{\tau}\right) E$.

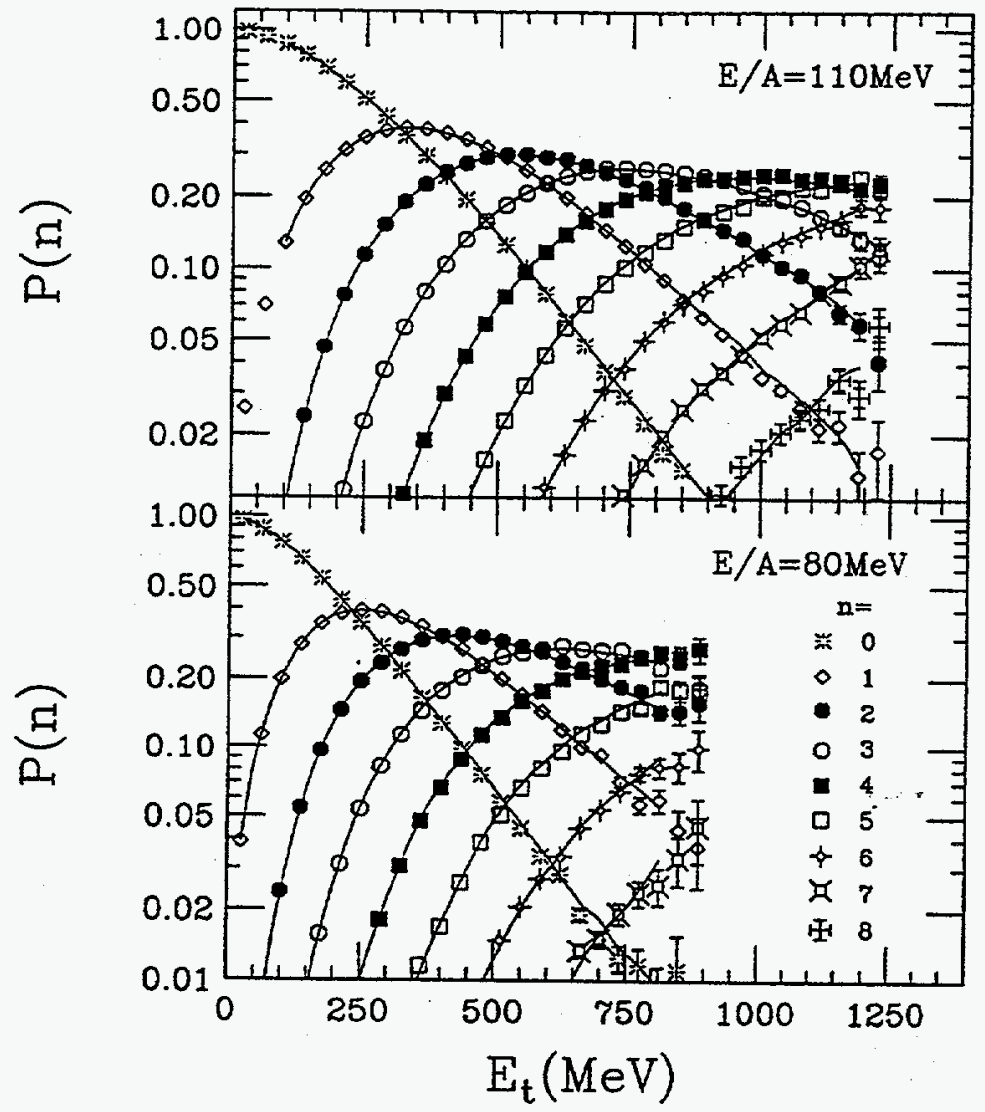

Fig. 6 . The calculated probability to emit $n$ intermediate mass fragments $(3 \leqq Z \leqq 20)$ as a function of $E_{t}$ for the reaction ${ }^{36} \mathrm{Ar}+$ ${ }^{197} \mathrm{Au}$ at $\mathrm{E} / \mathrm{A}=80$ (lower panel) and $110 \mathrm{MeV}$ (upper panel). For numbers of fragments $n=0-8, P(n)$ is calculated assuming a binomial distribution (see Eq. 9) with the values of $p$ shown in Fig. 6.
From Eqs. 10 and 11, we extract the elementary probability $p$ and $m$ from the mean and variance of the experimental multiplicity distributions $[39,40]$ for the ${ }^{36} \mathrm{Ar}+{ }^{197} \mathrm{Au}$ reactions at $E / A=80$ and $110 \mathrm{MeV}$.

In Fig. 5, we plot $1 / p$ vs. $E_{t}^{-1 / 2}$ for the fragment distributions using a $\log$ scale. If the probability $p$ is statistical, as given in Eq. (7), this plot ought to be linear[37] since $T \propto \sqrt{E}$. The linearity of this plot over two orders of magnitude is stunning, and leaves little doubt regarding the "thermal" nature of $p$.

The dramatic contraction of the time scale down to values close to the characteristic channel time shows that the onset of fragment-fragment or spectator-fragment interactions at high excitation energy is a natural consequence of Eqs. (7) and (8). The difference in slope for the two bombarding energies strongly suggests that the simple proportionality law between $E_{t}$ and $E$ is well satisfied.

The calculated excitation functions using the experimental values of $m$ and $p$ from the above procedure are shown if Fig. 6 with the experimental data[42]. There is an extraordinary quantitative agreement 
between the calculations and the experimental data.

Preliminary analysis of two additional experiments [43, 44] with different targetprojectile combinations and bombarding energies confirms the general applicability of this description. The rigorous applicability of the binomial distribution is very powerful evidence for the reducibility of the $n$-fold probabilities $P_{n}$ to the elementary binary probability $p$ and for the sequential structure of the multifragmentation event. The linearity of the extracted elementary binary probability plot (see Fig. 5) over two orders of magnitude is truly stunning, and indicates the thermal nature of the process at all excitation energies. The associated time-scale demonstrates the expected smooth contraction in time with increasing excitation energy without any indication of a new mechanism appearing at the highest energies.

We have tried to find alternative explanations for the binomial distributions with thermal probabilities. An obvious model is a chain of $m$ links with probability $p$ that any of the links is broken. The probability that $n$ links are broken is given by Eq. (9). This result is, of course, strictly dependent on the dimensionality of the model, and its relevance to multifragmentation is unclear. Nevertheless, it stresses again the fundamental reducibility of the multifragmentation probability to a binary breakup probability.

In summary:

1) The multifragment emission probability is rigorously binomial and is reducible to an elementary binary probability applied sequentially.

2) This binary elementary probability is "thermal", as demonstrated by its characteristic energy dependence.

3) The time scale of sequential emission contracts rapidly with increasing excitation energy as demanded by point 2). This contraction naturally explains the observed rapid onset of the fragment-fragment Coulomb interaction with increasing excitation energy and obviates the need for "simultaneous" multifragmentation as a distinct process.

4) The parameter $m$ could be truly a physical quantity rather than an artificial cutoff. It may indicate a dynamical time interval during which fragments are thermally emitted with high probability (transient expansion phase?)

\section{Acknowledgments}

This work was supported by the Director, Office of Energy Research, Office of High Energy and Nuclear Physics, Nuclear Physics Division of the US Department of Energy, under contract DE-AC03-76SF00098.

\section{References:}

[1] E. Wigner, Trans. Faraday Soc. 34, 29 (1938).

[2] H. A. Kramers, Physica 7, 284 (1940).

[3] P. Grange, J.-Q. Liu, and H. A. Weidenmüller, Phys. Rev. C27, 2063 (1983).

[4] P. Hänggi, P. Talkner, and M. Borkovec, Rev. Mod. Phys. 62, 251 (1990).

[5] L. G. Moretto, Nucl. Phys. A247, 211 (1975).

[6] M. A. McMahan, et al., Phys. Rev. Lett. 54, 1995 (1985).

[7] D. N. Delis, et al., Nucl. Phys. A534, 403 (1991).

[8] K. X. Jing, et al., Lawrence Berkeley Laboratoy LBL-to be published (1994).

[9] A. Gavron, et al., Phys. Rev. Lett. 47, 1255 (1981).

[10] E. Holub, et al., Phys. Rev. C 28, 252 (1983).

[11] D. J. Hinde, et al., Phys. Rev. C 45, 1229 (1992).

[12] D. Hilscher and H. Rossner, Ann. Phys. Fr. 17, 471-552 (1992).

[13] D. J. Hinde, et al., Nucl. Phys. A 452, 550 (1986).

[14] D. Hilscher, et al., Phys. Rev. Lett. 62, 1099 (1989). 
[15] E. Mordhorst, et al., Phys. Rev. C 43, 1991 (1991).

[16] L. G. Sobotka, et al., Phys. Rev. Lett. 51, 2187 (1983).

[17] L. G. Moretto and G. J. Wozniak, Prog. Part. \& Nucl. Phys.,Vol. 21, ed. 1988), p. 401.

[18] W. G. Lynch, in Ann. Rev. Nucl. Part. Sci, by Vol. 37, ed. 1987), p. 493.

[19] D. Guerreau, Formation and Decay of Hot Nuclei: The Experimental Situation ed. (Plenum Publishing Corp., 1989).

[20] H. H. Gutbrod, A. M. Poskanzer, and H. G. Ritter, Rep. Prog. Phys., Vol. 52, ed. 1989), p. 1267-328.

[21] D. H. E. Gross, Rep. Prog. Phys. , by Vol. 53, ed. 1990), p. 605.

[22] L. G. Moretto and G. J. Wozniak, Ann. Rev. Part. \& Nucl. Sci, by Vol. 43, ed. 1993), p. 379.

[23] J. Aichelin;Phys. Rep., by Vol. 202, ed. 1991), p. 233-360.

[24] B. Borderie, Ann. Phys. Fr. 17, 349 (1992).

[25] O. Schapiro and D. H. E. Gross, Hahn-Meitner-Institut, Berlin GmbH preprint (1994).

[26] T. Ethvignot, et al., Phys. Rev. 48C, 618 (1993).

[27] D. Fox, et al., Phys. Rev. C47, R421 (1993).

[28] E. Bauge, et al., Phys. Rev. Lett. 70, 3705 (1993).

[29] D. R. Bowman, et al., Phys. Rev. Lett. 70, 3534 (1993).

[30] T. C. Sangster, et al., Phys. Rev. C47, R2457 (1993).

[31] M. Abouffirassi, et al., LPC Caen preprint LPCC 94-02 (1994).

[32] A. Lleres, et al., ISN Grenoble ISN 94-33 (1994).

[33] M. Louvel and et. al., Phys. Lett. B 320, 221 (1994).

[34] R. J. Charity, et al., Nucl. Phys. A483, 371 (1988).

[35] J. Hüfner, in Phys. Rep. , by Vol. 125, ed. 1985), p. 129.

[36] J. P. Bondorf, et al., Nucl. Phys. A443, 321 (1985).

[37] L. G. Moretto, D. N. Delis, and G. J. Wozniak, Phys. Rev. Lett. 71, 3935 (1993).

[38] J. Pouliot, et al., Phys. Rev. C 48, 2514 (1993).

[39] L. Phair et. al, Phys. Lett. B 191, 7 (1992).

[40] L. Phair, Michigan State University, 1993.

[41] R. Bougault et al., LPC Caen LPCC 94-04 (1994).

[42] L. Phair et al., to be published (1994).

[43] K. Tso et al., to be published (1994).

[44] D. N. Delis et al., to be published (1994). 